\title{
LEAN MANAGEMENT - EIN MANAGEMENT FORM MIT ZUNEHMENDER RELEVANZ FÜR DEN TOURISMUS
}

\author{
Romualdas Ginevičius $^{1}$, Thomas Hausmann ${ }^{2}$, Schlomo Schafir ${ }^{3}$ \\ ${ }^{1}$ Vilnius Gediminas technische Universität, Saulètekio al. 11, LT-10223 Vilnius, Litauen \\ E-mail: ${ }^{1}$ romualdas.ginevicius@adm.vgtu.lt \\ ${ }^{2,3}$ Fachhochschule Stralsund, Zur Schwedenschanze 15, 18435 Stralsund, Germany \\ Empfangen 25-02-2007; angenommen 18-11-2007
}

\begin{abstract}
Auszug. Es kommt auf eine ganzheitliche Optimierung an. Ressourcen und Prozesse eines Unternehmens und seiner Zulieferer müssen so auf den Kunden-(Netto-)Nutzen ausgerichtet sein, dass Verschwendung vermieden werden kann. Das ist in industriellen Unternehmen schon lange bekannt. Der vorliegende Aufsatz geht der Frage nach, wie sich Lean Management in Touristik und Gastgewerbe umsetzen lässt.

Schlüsselwörter: Leitgedanken, Proaktives Denken, Sensitives Denken, Ganzheitliches Denken, Potentialdenken, Okonomisches Denken, Kerngedanke des Proaktiven Denkens, Kerngedanke des Sensitiven Denkens, Kerngedanke des Ganzheitlichen Denkens, Kerngedanke des Potentialdenkens, 10 Arbeitsprinzipien.
\end{abstract}

\section{LEAN MANAGEMENT - A FORM OF MANAGEMENT WITH INCREASING RELEVANCE FOR TOURISM}

\author{
Romualdas Ginevičius ${ }^{1}$, Thomas Hausmann ${ }^{2}$, Schlomo Schafir ${ }^{3}$ \\ ${ }^{1}$ Vilnius Gediminas Technical University, Sauletekio al. 11, 10223 Vilnius, Lithuania \\ E-mail: ${ }^{1}$ romualdas.ginevicius@adm.vgtu.lt \\ ${ }^{2,3}$ Stralsund University of Applied Sciences, Zur Schwedenschanze 15, 18435 Stralsund, Germany
}

Received 25 February 2007; accepted 18 November 2007

\begin{abstract}
Integral optimization matters the most. Resources and processes of a company and its suppliers have to be focused on customer-net benefit in such a way that wasting can be avoided. This has been known in industrial companies for a long time. The article investigates the question how lean management in tourism companies and hospitality industry can be adapted.
\end{abstract}

Keywords: central ideas, proactive thinking, sensitive thinking, integrated thinking, potential thinking, economical thinking, core idea of proactive thinking, core idea of sensitive thinking, core idea of integrated thinking, core idea of potential thinking, 10 work principles. 


\section{Einführung}

Das Lean Management-Konzept bringt in der Führung und Organisation industrieller Unternehmen einen prinzipiellen Fortschritt, dessen Erfolgsdimension nur vergleichbar ist mit dem Schritt von der handwerklich orientierten Produktion zum industriellen Massenproduktions-Konzept von F. W. Taylor und Henry Ford. Wie die Konzeptionen von Taylor und Ford die optimale Antwort auf die politisch-gesellschaftlichen, wirtschaftlichen und technischen Rahmenbedingungen des beginnenden 20. Jahrhunderts waren, ist das Lean Management-Konzept die überzeugende Antwort auf die Rahmenbedingungen in Markt, Technik und Gesellschaft am Anfang des 21. Jahrhunderts.

In beiden Fällen ging es nicht nur um bloße Innovationen in den Methoden, sondern um fundamentale Innovationen im Denken, in der Philosophie von Führung und Organisation industrieller Unternehmen (Pfeiffer, Weiss 1992: 1).

\section{Begriffsbestimmung}

Kaum ein Führungsthema hat jemals öffentliche Medien, Unternehmer und Gewerkschaften so bewegt wie Lean Management. Diese ursprünglich in Japan entwickelte Form der Unternehmensführung produziert Güter wie Dienstleistungen mit erheblich geringerem Aufwand in vorzüglicher und kundengerechter Qualität.

Lean Management repräsentiert ein Bündel von Prinzipien, Konzeptionen und Maßnahmen zur effektiven und effizienten Planung, Gestaltung und Kontrolle der gesamten Wertschöpfungskette eines Unternehmens.

Bösenberg/Metzen konkretisieren zum Begriff sehr treffend folgendes (Bösenberg, Metzen 1995: 7).

Lean Management ist ein komplexes System, welches das gesamte Unternehmen umfasst. Es stellt den Menschen in den Mittelpunkt des unternehmerischen Geschehens. Seine Elemente sind fundierte geistige Leitlinien, Arbeitsprinzipien mit neuen Organisations überlegungen, integrierende Strategien zur Lösung der zentralen Unternehmensaufgaben, wissenschaftlich-ingenieurmäßige Methoden sowie eine Reihe pragmatischer Arbeitswerkzeuge für Mitarbeiter.

„Leane“ Unternehmen kennzeichnet zusätzlich zur schlanken Produktion ein besonderes Verhältnis zu den Kunden, den Lieferanten, den Mitarbeiterinnen und Mitarbeitern sowie eine ungewöhnliche Finanzstrategie.

\section{Tourismusunternehmenspolitik als konzeptionelle Voraussetzung}

Ein Tourismusunternehmen kann nur dann gedeihen, wenn es in den Augen der Gäste, der Mitarbeiter und der allgemeinen Öffentlichkeit einen echten Nutzen erbringt.
Die Unternehmenspolitik regelt die Beziehungen innerhalb des Betriebs und zu den Marktpartnern. Sie enthält Ziele und Verhaltensgrundsätze (Dehoga 1992: 43).

Die Unternehmenspolitik ist somit dem Lean Management übergeordnet, denn bevor die Denk- und Grundsätze des Lean Management erörtern werden können, müssen die generellen Ziele und Verhaltensnormen (Unternehmensgrundsätze) zur Sicherung der Lebensund Entwicklungsfähigkeit des Unternehmens festgelegt werden, der allgemeine Kurs, auf dem sich das Unternehmen künftig entwickeln soll. Dies bezweckt, dass alle Unternehmensmitglieder am „selben Strick“ in dieselbe Richtung ziehen (Kaspar 1995: 61).

Die Zielausrichtung der Unternehmenspolitik spricht eine Grundorientierung des Unternehmens an, die bei touristischen Unternehmen und insbesondere bei Tourismusorganisationen mit einer Non-profitAusrichtung (regionale Tourismusverbände, örtliche Verkehrsbüros/Tourist Information) kaum klar festgelegt werden kann, sondern „sowohl-als-auch“ Ziele enthält, womit in vielen Fällen eine Anforderung an die Grundsätze der Unternehmenspolitik, nämlich die Konsistenz, verletzt wird. Wie im Einleitungsabsatz zu diesem 2. Gliederungspunkt festgestellt, kann ein touristisches Unternehmen nur dann gedeihen, wenn sie in den Augen der Gäste, der Mitarbeiter, der Eigentümer, der Geschäftspartner und der allgemeinen Öffentlichkeit einen echten Nutzen erbringt. Eine solche pluralistisch gesellschaftsorientierte Zielausrichtung (sozial- und umweltverträglich) mit langfristiger Nutzenstiftung (regional- und lokalwirtschaftlicher Ansatz) entspricht dem „Stakeholder-Ansatz“, im Gegensatz zum „ShareholderAnsatz" mit kurzfristig eindimensionaler Ausrichtung auf die Interessen der Eigentümer.

Die Unternehmenspolitik enthält übergeordnete und sektorielle Ziele als Richtgrößen. Zu den übergeordneten Unternehmenszielen gehören qualitative Ziele wie Erhaltung der unternehmerischen Selbständigkeit oder Ausrichtung auf Sozial- und Umweltverträglichkeit, und quantitative Ziele wie Sicherstellung der Rentabilität, Festlegung von Kapazitäten und Aufrechterhaltung eines vorteilhaften Preis- / Leistungsverhältnisses. Sie haben unternehmensweite Gültigkeit.

Sektorielle Unternehmensziele betreffen einzelne Tätigkeitsbereiche wie Mitarbeiterschulung, optimale Organisationsstruktur oder Marketing und wirken direkt nur auf das entsprechende strategische Geschäftsfeld beziehungsweise die entsprechende Unternehmensfunktion.

\section{Zentrale Denkansätze von Lean Management}

Lean Management will die zukunftsorientiete, intelligente und konsequente Weiterentwicklung der unternehmenseigenen Stärken und Chancen. Die „Fitness-Tour“ zum 
schlanken Unternehmen endet nie. Im Mittelpunkt steht kein „überreiztes Abmagern“, sondern eine integrierte, abgestimmte und ganzheitliche Schlankheitskur: Offensiv und behutsam, traditionell und anpassungsbereit, konsequent und flexibel, kräftig und schlank (Schaetzing 2002: 11).

\section{Lean = schlank, d.h. sorgfältiger Umgang mit den Ressourcen}

Damit kommt das Lean Management dem Management touristischer Unternehmen entgegen, wenn an die Umweltsensibilität touristischer Landschaften oder an die Knappheit der menschlichen und finanziellen Ressourcen touristischer Unternehmen gedacht wird. Auch die relative Größe touristischer Unternehmen, etwa die kleinund mittelbetriebliche Struktur der Beherbergungs- und Verpflegungsbetriebe westeuropäischer Staaten, entsprechen kleinen Managementebenen leaner Unternehmen mit flachen Führungspyramiden Abb. 1. Vorteilhaft für die Anwendung des Lean Management im Tourismus erweisen sich Einzelverantwortung und Entscheidungsfreiheit im Zusammenhang mit den vielen Schnittstellen Gast/ Kunde - Mitarbeiter.

Zentrale Denkansätze des Lean Management in den Köpfen aller Mitarbeiter und Mitwirkenden sichern die Gemeinsamkeit und die Selbständigkeit der einzelnen Unternehmensinhalte. Vorstand bzw. Führung wie Mitarbeiter lassen sich bei ihrem Tun von den identischen Leitbildern inspirieren (Bösenberg, Metzen 1995: 37).

\section{Die fünf Leitgedanken des Lean Management}

Bötzenberg/Metzen erklären als Philosophie des „schlanken“ Denkens fünf Leitgedanken:

\section{a) Proaktives Denken}

Künftige Handlungen werden vorausschauend initiativ durchdacht und gestaltet.

\section{b) Sensitives Denken}

Mit allen verfügbaren Sensoren die Umwelt erfassen und anpassungsbereit darauf reagieren.

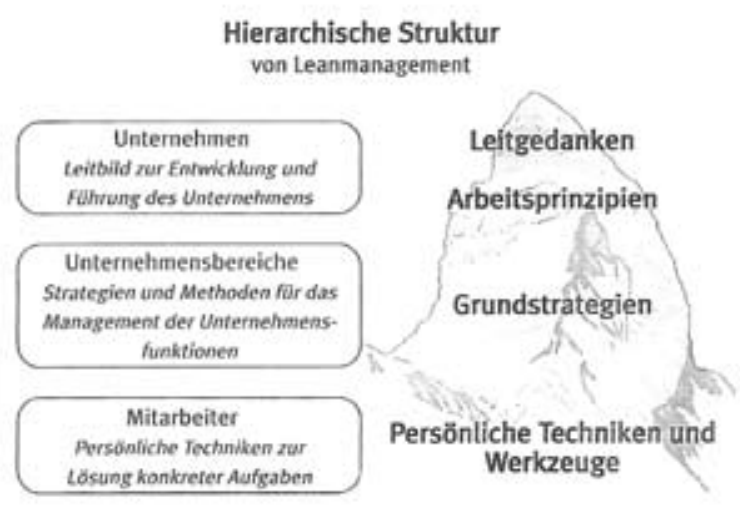

Abb. 1. Hierarchische Struktur von Lean Management (Bösenberg, Metzen 1995: 39)

\section{c) Ganzheitliches Denken}

Die Wirkung auf das Ganze bedenken und Mut zur Komplexität beweisen.

\section{d) Potentialdenken}

Alle verfügbaren Ressourcen erschließen und nutzen.

\section{e) Ökonomisches Denken}

Vermeidung jeder Verschwendung, sparsam wirtschaften.

Diese fünf Leitgedanken werden von den Autoren, wie nachfolgend wiedergegeben, noch konkretisiert. Sie stellen dem Denken der Manager Leitlinien, mit denen erfolgreich geführt werden kann und entsprechen weitgehend der in der normativen Ebene des Managements enthaltenen Management-Philosophie nach Kaspar (Kaspar 1995: 58 ff).

\section{zu a) Kerngedanke des proaktiven Denkens}

\section{Agieren statt reagieren}

Dieser Kerngedanke sichert den Erfolg, wird aber oft nicht beherzigt, zumal er besondere Anstrengungen, ein sich Lösen vom Erreichten, bedeutet. Voraus-schauend die Prozesse unter Kontrolle bringen, damit unliebsame Überraschungen vermieden werden können.

\section{Alle Handlungen umfassend vorbereiten}

Alle erkennbaren Probleme frühzeitig lösen und nicht auf morgen verschieben, um Zeit und Kapazität für aktuelle versteckte Probleme zu gewinnen.

\section{Prozess- statt Ergebnisorientierung}

Das Ergebnis ist die Folge des vorangegangenen Prozesses. Wenn wir der Prozessabwicklung nicht die nötige Sorgfalt auch im Sinne eines vorausschauenden Handelns angedeihen lassen, können wir nicht mit einem befriedigenden Ergebnis rechnen.

Für das proaktive Denken kann folgende Checkliste vorgesehen werden Abb. 2.

Wie die Autoren richtig bemerken, setzt Proaktivität

\begin{tabular}{lcc}
\hline Merkmal & Proaktives Denken & Reaktives Denken \\
\hline Führungsverhalten & leistungsbetont & machtbetont \\
\hline Führungsideale & Krisenvermeider & Krisenmanager \\
\hline Aufgabenannahme & initiativ & abwartend \\
\hline Aufgabenerledigung & gestaltend & ausführend \\
\hline Zukunftssicht & optimistisch & pessimistisch \\
\hline $\begin{array}{l}\text { Übernahme von } \\
\text { Verantwortung }\end{array}$ & bereitwillig & widerstrebend \\
\hline $\begin{array}{l}\text { Unternehmensklima } \\
\text { Auftreten überra- } \\
\text { schender Probleme }\end{array}$ & selten & Aktionismus \\
\hline
\end{tabular}

Abb. 2. Checkliste für das proaktive Denken (Dettmer et al. 1999: 60) 
mehr auf die dauernde Weiterentwicklung der eigenen Stärken und Motive als auf das kurzfristige Erlangen von Überlegenheit gegenüber Wettbewerbern. Dazu gehört auch die Gefahr, die Erfolgsfaktoren des Konkurrenten zu imitieren, uns indessen nicht dieselben Human - und Finanzressourcen sowie andere Vorteile, wie Standort oder Image zur Verfügung stehen.

\section{zu b) Kerngedanke des Sensitiven Denkens}

\section{Informationsoffenheit nach innen und außen}

„Wissen ist Macht" sollte auch bei allen Mitarbeitern gelten. Das sog. Know how sollte insbesondere innerhalb des Betriebes weitergegeben werden, damit etwa frühere Fehler vermieden werden können. Zweckdienliche Informationen sollten aber auch nach außen vermittelt werden, zumal sie werbewirksam sind.

Weitere Kerngedanken zum Sensitiven Denken nennen Bösenberg/Metzen:

- Mit allen Sinnen Informationen aufnehmen.

- Gefühle und Stimmungen neben Fakten als Entscheidungsfaktoren akzeptieren.

- „Störungen“ von Außen sind Anregungen zur Entwicklung.

\begin{tabular}{|l|l|l|}
\hline Merkmal & Sensitiv & Repressiv \\
\hline Informationsaufnahme & offen & gesteuert \\
\hline Information Vorgesetzter & offen & glorifizierend \\
\hline Kommunikation & vernetzt & 1-Weg \\
\hline Anweisungen & Vorschlag & Befehl \\
\hline Lernfähigkeit & hoch & gering \\
\hline Veränderungsbereitschaft & hoch & gering \\
\hline Reaktion auf Fehler & verbessernd & bestrafend \\
\hline Reaktion auf Kritik & zuhörend & abwehrend \\
\hline Eigenbild & $\begin{array}{l}\text { realistisch, } \\
\text { kritisch }\end{array}$ & glorifizierend \\
\hline
\end{tabular}

Abb. 3. Checkliste Sensitives Denken (Dettmer et al. 1999: 61)

\section{zu c) Kerngedanke des Ganzheitlichen Denkens}

- Ganzheitlichkeit erkennt mehr Probleme am Horizont.

- Das die heutigen Marktgesetze beherrschende Prinzip der Globalisierung und Liberalisierung verlangt ganzheitliches Denken. Nur die Ganzheitlichkeit sozialer Systeme wie das Unternehmen garantiert Mitdenken, Eigenverantwortung, Zielstrebigkeit und aktiven „Überlebenswillen“ (Bösenberg, Metzen 1995: 52). Dies bedeutet für die Autoren im weitern:

- Der Wert der eigenen Handlung richtet sich nach dem Nutzen für das Gesamtsystem (Unternehmen).

- Systeme rächen sich, wenn der eigene Vorteil nicht zu einem Vorteil für das System (Unternehmen) führt.

- Räumliches und zeitliches Ganzheitsdenken.

- Komplexe Prozesse entwickeln Eigendynamik und sind nicht von einer Person allein zu kontrollieren.

- Die Kräfte des Ganzen für die eigenen Handlungen mitbenutzen. Abb. 3.

- Netzwerke statt dualer Beziehungen.

zu d) Kerngedanke des Potentialdenkens Erschliessung aller Ressourcen

Es ist immer wieder auf die Bedeutung der humanen und finanziellen Ressourcen für die Überlebensfähigkeit des Unternehmens hinzuweisen. Bekannt ist auch die Forderung nach Humanisierung der Arbeitswelt. Diese Überlegungen gehen u.a. in diesen Kerngedanken der beiden Autoren ein:

- Mitarbeiter, Lieferanten, Kunden, Wettbewerber als Ressourcen nutzen.

- Aufhebung der Trennung von Denken und Arbeiten: Alle Mitarbeiter, nicht nur die Vorgesetzten zum Mitdenken animieren, damit wird auch die Motivation des Einzelnen gefördert.

- Gleichgerichtete Interessen schaffen.

- Überall die Frage nach ungenutzten Ressourcen stellen.

- Unter- und Überforderungen vermeiden.

- Gemeinsam erzielten Nutzen fair teilen. Dieser Kerngedanke scheint schwierig zu verwirklichen und ist oft Gegenstand von Auseinandersetzungen zwischen den Mitarbeitern, obwohl doch eine faire Teilung Arbeitsmoral und -motivation wesentlich zu heben vermag.

\section{zu e) Kerngedanke des Ökonomischen Denkens}

Ein wichtiges, von "Lean-Unternehmen“ verfolgtes Prinzip besteht in der Vermeidung von Verschwendung. Bösenberg/Metzen schließen dieses Prinzip allgemein in „Kerngedanken Ökonomisches Denken“ ein.

- Neudefinition von Verschwendung:

Alle nichtwertschöpfenden Tätigkeiten, Lager und Puffer entfallen lassen. Die besten Leute an Stellen mit der höchsten Wertschöpfung.

- Qualität geplant erzeugen, kein Ausschuss und Nacharbeit. Verdeckten Aufwand aufspüren.

- Sparsam nach innen, aber nicht vor Kunde/Gast.

Kunden- bzw. Gastnutzen der Leistung bzw. des Produkts so hoch wie möglich. Hohe Präsenz und ordentliches Auftreten im Markt. 
- Konflikte bedeuten Kosten.

Harmonisierung der Interessen mit allen Partnern (Mitarbeiter, Lieferanten, Kunden, Kapitalgeber, Staat).

\section{Die 10 Arbeitsprinzipien des Lean Management im Tourismus}

Arbeits- und Organisationsprinzipien stellen eine Übersetzung der allgemeinen Leitgedanken in die "Sprache" der konkreten Arbeitsorganisation dar.

Die Arbeitsprinzipien - wie die Leitgedanken des Lean Management erfüllen die Funktion der delegationsunabhängigen Mitarbeiter- und Unternehmensführung auf den unterschiedlichen Unternehmensebenen in unterschiedlichen Dimensionen. Sie liefern Problemlösungsprinzipien in neuen oder unsicheren Situationen und sagen, wie Entscheidungen umzusetzen sind.

Dabei werden 10 Arbeitsprinzipien unterschieden.

\section{Arbeitsprinzip Nr. 1: Gruppe, Team}

Die Aufgaben werden in der Gruppe oder im Team erledigt. Der Konsenzgedanke ist bei der Lösung der Aufgabe dominant, interner Wettbewerb wird vermieden.

\section{Arbeitsprinzip Nr. 2: Eigenverantwortung}

Jede Tätigkeit wird in Eigenverantwortung durchgeführt. Den Rahmen dazu bilden die Standards, die für jede Tätigkeit erstellt werden. Kann die geforderte Qualität nicht eingehalten werden, wird der Arbeitsfluss unterbrochen und Hilfe angefordert.

\section{Arbeitsprinzip Nr. 3: Feedback}

Alle Aktivitäten, vom einzelnen bis zum kompletten Funktionsbereich, werden von einem außergewöhnlich intensiven Feedback (Rückkoppelung) begleitet. Die Reaktionen von Außenwelt, System oder Anlagen dienen zur Steuerung des eigenen Handelns.

\section{Arbeitsprinzip Nr. 4: Kunden/Gastorientierung}

Alle Aktivitäten sind streng auf den Kunden orientiert. Die Wünsche des Kunden/Gastes haben Priorität im Unternehmen.

\section{Arbeitsprinzip Nr. 5: Wertschöpfung hat Priorität}

Die wertschöpfenden Tätigkeiten haben erste Priorität im Unternehmen. Das gilt für alle verfügbaren Ressourcen.

\section{Arbeitsprinzip Nr. 6: Standardisierung}

Formalisierung und Standardisierung der Arbeitsgänge durch einfache schriftliche und bildliche Darstellungen.

\section{Arbeitsprinzip Nr. 7: Ständige Verbesserung}

Die ständige Verbesserung aller Leistungsprozesse bestimmt das tägliche Denken. Es gibt keine entgültigen Ziele, sondern nur Schritte in die richtige Richtung.

Arbeitsprinzip Nr. 8: Sofortige Fehlerabstellung an der Wurzel
Jeder Fehler wird als Störung des Prozesses angesehen, dem bis auf die eigentliche Ursache nachzugehen ist.

Arbeitsprinzip Nr. 9: Vorausdenken, Vorausplanen

Nicht die erfolgreiche Reaktion, sondern die Vermeidung künftiger Probleme gilt als

Ideal. Das Denken erfolgt wie bei einem Schachspieler über mehrere Züge im voraus.

Arbeitsprinzip Nr. 10: Kleine, beherrschte Schritte

Die Entwicklung erfolgt in kleinen, beherrschten Schritten. Das Feedback auf jeden Schritt steuert den nächsten. Die Geschwindigkeit wird durch die schnelle Folge der Schritte erhöht (Dettmer et al. 1999: 70 f.).

Weitere Prinzipien des Lean Management sind Integriertheit, Systematik, Interdisziplinarität, Permanenz, Konsequenz und Perfektion. Als Optimierungskriterien gelten auf der Sachebene Qualität, Zeit, Flexibilität und Produktivität, auf der Wertebene Kosten, Erlöse und Rentabilität.

\section{Die sechs Grundstufen des Lean Management im Tourismus}

Allgemein ausgedrückt, ist das strategische Management auf den Aufbau, die Pflege und die Ausbeutung von Erfolgspotentialen gerichtet. Es orientiert sich an längerfristigen Zielen und definiert in Form von Konzepten die dispositiven Handlungsspielräume (Bleicher 2004: 54).

Die sechs „leanen“ Grundstrategien stellen Musterlösungen für die wichtigsten „internen“ Aufgaben des Unternehmens dar:

Produktion zu marktgerechten Kosten in kundengerechter Qualität und für einen wettbewerbsgerechten Zeitraum; Einstellung auf Markt und Kunden/Gast, förderliche Kapitalverwendung und schließlich Gestaltung der Beziehungen zu Mitarbeitern und Marktpartnern.

Unternehmen funktionieren dann hervorragend, wenn die sechs Grundstrategien laufend umgesetzt werden, als gemeinsames Strategiebündel (Dettmer et al. 1999: 63).

\subsection{Kunden- bzw. gastorientierte schlanke Leistungserstellung}

Für leistungsanbietende Unternehmen, wie im Tourismus vertreten, ist das schlanke Angebot ebenfalls von entscheidender Bedeutung. Für viele Unternehmen gilt das sog. Just in Time als Strategie, d.h. die begehrte Leistung sofort anbieten zu können. Beispielsweise können tiefgekühlte Fertiggerichte oder Halbgerichte, die durch MikroGrillapparate in Sekundenschnelle angerichtet werden können, Just in Time dem Gast vorgesetzt werden. Ein richtig verstandenes Just in Time-System kann die z.T. kostspielige Lagerhaltung (z. B. Getränke) beschränken. Durch eine richtig verstandene Personaleinteilung, welche die unterschiedlichen Frequenzen der Gäste berück- 
sichtigt, können Personalkosten eingespart werden. Durch Ablaufzeitstudien der Arbeitsprozesse und deren Organisation können Arbeitszeiten verkürzt werden, ohne dass dabei die Qualität leiden muss.

Just in Time heißt die richtige Leistung zum richtigen Zeitpunkt in der richtigen Qualität erbringen. Eine konsequente Just in Time-Strategie betrifft die ganze Wertschöpfungskette, ermöglicht eine bessere Integration der einzelnen Teile und deckt dabei leistungshemmende Fehler oder Schwierigkeiten auf.

\subsection{Unternehmensqualität in allen Bereichen}

Qualität ist auch im Tourismus zu einem erfolgversprechenden Element des Angebotes geworden. Zunehmender Wettbewerb unter einer stark wachsenden Zahl von Reisedestinationen sowie die steigenden Erwartungen und Ansprüche der Reisenden bestimmen immer mehr das Qualitätsniveau touristischer Leistungen. Qualität wird im Tourismus immer mehr zu einem konkurrenzrelevanten Faktor, wobei im Vordergrund das Qualitätsdenken und -bewusstsein der Mitarbeiter steht, basierend auf einem guten Aus- und Weiterbildungssystem.

Die Bedeutung der Qualität wird im Begriff Quality Management - QM - klar. Demnach hat QM zum Gegenstand, alle für den Kunden wesentlichen Anforderungen bezogen auf die angebotene Leistungsqualität des Marktangebotes des Unternehmens und die Kontaktqualität in der Kommunikation mit dem Kunden zu erfüllen. Voraussetzung hiefür ist eine Führungs- und Steuerungsqualität im Unternehmen (Hopfenbeck 2002: 570).

QM ist somit ein Management-Konzept zur Umsetzung der Qualitätspolitik in den betrieblichen Alltag. QM wird von Gardini als eine alle Mitarbeiter umfassende, langfristige Unternehmensphilosophie zur Durchsetzung des Qualitätsgedankens auf allen Ebenen des Unternehmens bezeichnet (Gardini 1994: 73).

Oft wird vom sog. 5-Q-Modell des Qualitätsmanagements für Dienstleistungen gesprochen (vgl. Abb. 4).

Auf der Basis des QM-Konzeptes umfasst das 5Q-Modell fünf Bausteine, die den Rahmen für die Implementierung eines QM-Konzeptes bilden:

- Wertorientierung;

- Kundenorientierung;

- Personalorientierung;

- Optimierung und Restrukturierung des Qualitätssystems ;

- Kosten - und qualitätsorientiertes Controlling.

Gemäss Bösenberg/Metzen bestehen die Kerngedanken des Quality Management in folgenden Punkten (Bösenberg, Metzen 1995: 154):

a) Qualität ist ein bedeutsamer strategischer
Erfolgsfaktor und damit eine Unternehmensaufgabe.

- Aufgabe für jeden und nicht nur Spezialisten.

- Jeder Manager muss Qualitätsmanagement betreiben und verantworten.

- Jeder ist für die Qualität seiner Arbeit verantwortlich - Fehler anderer werden mit beseitigt und abgestellt.

b) Qualität aus Kundensicht betrachten - der Kunde bestimmt Qualitätskriterien und -maßstäbe.

- Qualität aus Kundensicht messbar und planbar machen.

- Qualität aus Kundensicht umfasst objektive und subjektive Faktoren.

- Emotionale Faktoren und Sinnesempfindungen ein beziehen.

c) Qualität ist relativ zum Wettbewerb, zu Erwartungen und Erfahrungen

- Die gleiche technische Qualität kann bei schwachen Märkten hervorragend und in starken Märkten ungenügend sein.

- Qualitätsurteile sind dynamisch; was gestern hervor- ragend war, kann heute Mittelmass und morgen ungenügend sein.

d) Qualitätsmanagement versucht, die Fehler immer weiter im voraus abzustellen

- Prozessregelung ersetzt Ergebniskontrolle (Inspektion).

- Prozessoptimierung und sichere Prozessplanung ersetzt Prozessregelung.

- Qualitätsplanung erkennt und eliminiert mögliche Fehlerursachen im voraus.

- Quality Function Deployment (QFD) setzt Kundenanforderungen in technische Merkmale um.

e) Es gibt keine Qualitätskosten, aber erhebliche Fehlleistungskosten

- Offene Fehlleistungskosten sind Gewährleistung, Prüfung des Sortiments, Nacharbeit.

- Versteckte Fehlleistungskosten betragen ein Vielfaches der offenen Kosten (geringe Kunden/Gastloyalität, überdimensionierte Lager, aufwendige, unflexible Fertigungssteuerung, unnötige Verwaltungs- und Transportvorgänge, Managementbedarf für Chaos und Störungen aller Art).

Mit dem Quality Management wird die Qualität zur zentralen Größe der Unternehmenstätigkeit. Angefangen bei der Qualitätsplanung und des Setzens von Qualitätszielen, über die Qualitätsfertigung und die Qualitätsprüfung bis hin zur Qualitätsanalyse umfasst es den ganzen Leistungserstellungsprozess. Romeiss-Stracke unterscheidet folgende Komponenten der touristischen Qualität (Abb. 4): 


\begin{tabular}{|c|c|c|c|c|c|}
\hline \multicolumn{6}{|c|}{ Qualität im Tourismus } \\
\hline \multicolumn{2}{|c|}{ Materielle Qualität = Hardware } & \multicolumn{2}{|c|}{ Natürliche Qualität = Umwelt } & \multicolumn{2}{|c|}{ Immaterielle Qualität = Software } \\
\hline Funktion & Ästhetik & $\begin{array}{l}\text { natürliche } \\
\text { Lebensgrundlagen }\end{array}$ & $\begin{array}{l}\text { gebaute } \\
\text { Umwelt }\end{array}$ & Servicegeist & $\begin{array}{l}\text { Qualität der } \\
\text { Information }\end{array}$ \\
\hline $\begin{array}{l}\text { einwandfreie } \\
\text { Funktionsweise } \\
\text { als Selbstver- } \\
\text { ständlichkeit }\end{array}$ & $\begin{array}{l}\text { Ortsbild } \\
\text { Innenarchi- } \\
\text { tektur } \\
\text { Dekoration } \\
\text { Luft, Licht } \\
\text { Raumklima }\end{array}$ & $\begin{array}{l}\text { Wasser } \\
\text { Luft } \\
\text { Boden } \\
\text { Fauna } \\
\text { Flora }\end{array}$ & Architektur & $\begin{array}{l}\text { Hilfsbereitschaft } \\
\text { Freundlich- } \\
\text { keit, Gast- } \\
\text { freundschaft } \\
\text { Ferienkultur }\end{array}$ & $\begin{array}{l}\text { aktuell } \\
\text { schnell } \\
\text { sicher } \\
\text { umfassend } \\
\text { bequem }\end{array}$ \\
\hline
\end{tabular}

Abb. 4. Qualität im Tourismus (Romeiss-Stracke 1995: 16)

Im Tourismus sind in letzter Zeit zur Hebung der Qualität, insbesondere bezogen auf die Umweltqualität, verschiedene Gütesiegel, wie der Blaue Umweltengel, der Grüne Koffer gestaltet worden (Hausmann 2006: 397).

Qualitätswettbewerbe und Qualitätspreise haben die Aufgabe, das Bewusstsein der Branche und auch das der Öffentlichkeit für die jeweiligen Qualitätsaspekte zu schärfen (Romeiss-Stracke 1995: 17). Für die prämierten Unternehmen haben solche Preise nachweislich unschätzbare Vorteile, z.B.

- Imagegewinn und Steigerung des Bekanntheits-grades;

- Steigerung der Glaubwürdigkeit des Unternehmens;

- der Name, die Marke des Unternehmens wird zum Inbegriff für Qualität;

- die Position des Unternehmens am Markt festigt sich, die Gewinne steigen wegen erhöhter Nachfrage;

- der Stammkundenanteil erhöht sich;

- die Personalfluktuation verringert sich, weil die Mitarbeiter zufrieden sind;

- Kundenbeschwerden nehmen ab, die Kundenzufriedenheit wird deutlich höher.

Entsprechend der Devise „Quality never happens by itself“, muss Qualität organisiert und geführt werden. Um dies zu erreichen, muss ein Unternehmen ein Qualitätssystem entwickeln und einführen. Dementsprechend ist eine internationale Normenreihe ISO (ISO = International
Organization for Standarization) eine Dachgesellschaft von über 60 nationalen Normenausschüssen) ausgearbeitet worden. Mit einer „Norm“ kann, indem die Erfüllung ihrer Anforderungen zertifiziert (d. h. durch eine neutrale Zertifierungsgesellschaft bestätigt) wird, eine bestimmte Leistungsfähigkeit mit einem Audit nachgewiesen werden.

Für die uns interessierenden Dienstleistungen ist ISO 9000 maßgebend:

Das grundsätzliche Anliegen der Serie, deren darin beschriebenen Modelle nahezu alle Bereiche im Unternehmen berührt, ist die Abb. 5.

- Schaffung einer Aufbau- und Ablauforganisation zu Gewährleistung einer effizienten Qualitätszielerreichung;

- Einbeziehung und Qualifikation aller Mitarbeiter;

- Regelung von Zuständigkeiten, Verantwortung und Befugnissen;

- Dokumentationspflicht für Regelungen und Ergebnisse (damit Nachweis der Erfüllung vertraglicher Forderungen an das QM-System nach außen und nach innen, Transparenz der Abläufe);

- Berichtspflicht bis zur höchsten Leitungsebene;

- Beherrschung von Risiken und Wirtschaftlichkeit;

- Einrichtung vorbeugender Maßnahmen zur Vermeidung von Qualitätsproblemen;

- Durchführung von Audits (Review) (Hopfenbeck 2002: 534).
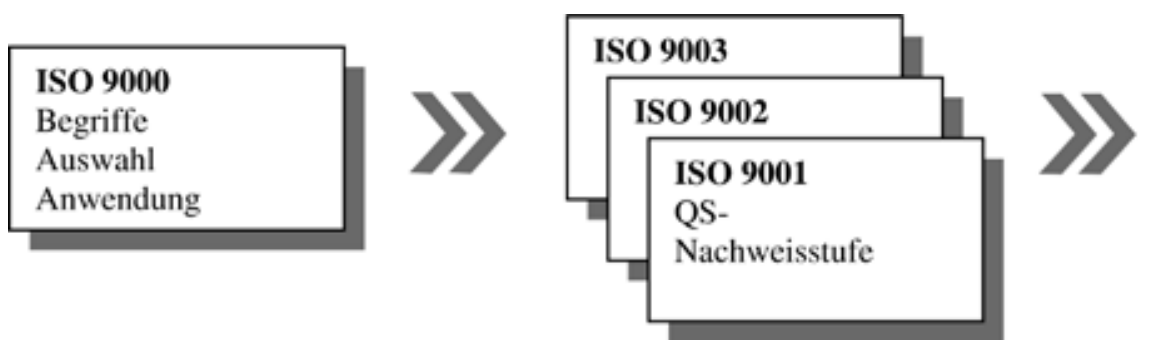

ISO 9004

QS-

Elemente

Richtlinien 
6.3. Schnelle, sichere Entwicklung und Einführung neuer Leistungen

Zeit ist nicht nur Geld, sondern bedeutet auch einen Erfolgsfaktor: der Erste auf dem Markt hat entscheidende Vorteile. In der Fachsprache wird das simultane Abarbeiten von Aufträgen Simultaneous Engineering genannt:

Entwicklungszeit als entscheidender Wettbewerbsfaktor. Der Erste auf dem Markt hat entscheidende Vorteile: bessere Preise, am Anfang, beste Chancen zur Marktführerschaft, beste Chancen, das System zu bestimmen. Beispiele aus dem Tourismus: neue Reisedestinationen mit besonderen Attraktionen, neuartige Hotel- und Gaststättentypen, die dem allgemeinen Nachfragetrend entsprechen oder einen solchen begründen.

- Zeit sparen durch parallele statt sequentielle Aufgabenerledigungbzw. Arbeitsabläufe wird insbesondere durch Teamarbeit ermöglicht.

In zeitkritischen Prozessen Trennung der Aufgaben in Kernarbeiten, vorbereitende und begleitende Tätigkeiten dabei ist die Frage der Auslagerung von Teilarbeiten gestellt, wie Gästewäsche, Zimmerreinigung und sportliche Begleitung der Gäste im Hotel; Auslagerung der Speiseherstellung bei Kantinen, Hotelund Restaurantketten, Arbeitsteilung zwischen dem Reiseveranstalter und dem Reisemittler usw.

\subsection{Kunden/Gäste gewinnen und erhalten}

Proaktives Denken wurde vorstehend als vorausschauend, initiativ durchdacht geschildert. Proaktives Marketing setzt auf langfristige und vertrauensvolle Zusammenarbeit zwischen Unternehmen und Kunden/Gäste. Auf diese Art der offensiven Gästebindung liegt der Schwerpunkt des Proaktiven Marketing nach Bösenberg/Metzen bei der Schaffung von substantiellen und anhaltenden Wettbewerbsvorteilen. Zukünftige Leistungsfähigkeit als Dienstleister für die Gäste ist wichtiger als kurzfristiger Erfolg.

Die Kerngedanken des Proaktiven Marketing im Tourismus sind nach Bösenberg/Metzen:

- Der loyale Kunde/Gast ist der beste Kunde/Gast Den Kunden/Gast so zufrieden machen, dass er wieder kauft. Den Kunden/Gast kompetent und ehrlich beraten. Kundenzufriedenheit ist messbar und planbar.

- Marketing ist keine Vertriebs-, sondern in erster Linie eine Unternehmensaufgabe. Jeder Manager betreibt auch Marketingmanagement. Direktkontakt zwischen Kunde/Gast und Unternehmen. Den Kunden/Gast aktiv in das Unternehmen einbeziehen. Im Markt leben, der Markt und mein Unternehmen sind eins.

- Kundenorientierung statt Produkt-/ Produktionsorientierung. Alle Unternehmensmaßstäbe aus
Kunden/ Gästesicht Kundenorientiert produzieren (Just in Time).

- Ich gehe zum Kunden/Gast statt: Der Kunde/Gast kommt zu mir. Konkrete statt abstrakte Kunden/ Gäste; auch versteckte Probleme des Kunden/Gastes erkennen und lösen.

- Markt und Produktion gestaltend synchronisieren. Marktschwächen aktiv ausgleichen oder (Kaspar 1995: 177 ff.).

\subsection{Wachstmus- und Eroberungsfähigkeit}

Die fünfte Grundstufe des Lean Management ist der strategische Kapitaleinsatz. Kapiteal gezielt einsetzenbedeutet knappe Ausstattung laufender Geschäfte und massiver Einsatz bei strategischen Projekten. Diese müssen sich nicht kurzfristig amortisieren, aber ein logischer und attraktiver Baustein in der Unternechmensentwicklung sein.

Auch in immaterielle Güter wie Mitarbeiter-Knowhow und Firmenimage ist zu investieren.

Weiterhin ist Vertrauen zwischen Kapitalgeber und Kapitalnehmer zu schaffen, denn Vertrauen zieht günstiges Kapital an.

6.6. Unternehmen in Gesellschaft harmo- nisch einbinden

Im Innen- wie Außenverhältnis eines touristischen Unternehmens ist zu betrachten, dass Konflikte teuer und aufwendig sind. Konflikte vermeiden durch Kooperation ist daher das Motto; das Unternehmen ist in die gesellschaftliche und wirtschaftliche Umwelt aktiv einzubeziehen (das Unernehmen als Familie betrachten).

Weiterhin sollte die volle Nutzung der Ressourcen von Lieferanten, Kunden, Mitarbeitern und Kapitalgebern Maßgabe sein.

\section{Controlling und Benchmarking als flankierende} Instrumente des Lean Management im Tourismus

Auch wenn das Lean Management mit dem Gebot eines dauernden und vorausschauenden Wirken verbunden ist, darf dabei das Controlling nicht übersehen werden.

Controlling ist bekanntlich modernes Konzept der Unternehmenssteuerung, das die Funktionen Information, Analyse/ Kontrolle, Planung und Steuerung einschließt.

Zwar konzentriert sich das Controlling hauptsachlich auf den finanziellen Bereich eines Unternehmens, es sollte aber als umfassenderes Managementsystem der ständigen Überprüfung der gesamten Unternehmenstätigkeit dienen (z. B. Marketing-Controlling, OrganisationsControlling usw.) Grenzen werden dem Konzept des Controlling erst dort gesetzt, wo Soll- und Ist- Werte nicht genau definierbar und messbar sind mit der 
Aufgabe der Unternehmenssteuerung ist das controlling zukunftsorient, zumal es die Realisierung der vordefinierten Zielgrößen überwacht und bei Abweichungen eingreift. Die Vergangenheit wird vom Finanz- und Rechnungswesen systematisch aufgezeigt und interessiert das Controlling nur insoweit, als die Vergangenheit Steuerungsimpulse für die Zukunft liefert.

Ein weiteres auch für das Lean Management wirksames Instrument bildet das Benchmarking.

Benchmarking oder „Lernen von den Besten“ wurde in den USA in den 80er Jahren entwickelt und in den Folgejahren von namhaften Unternehmen erfolgreich angewendet.

Hillen definiert Benchmarking „als eine strukturierte Methode zur Aufdeckung von eigenen Leistungslücken durch Vergleiche mit Bestleistungen (Benchmarks) mit dem Ziel, durch die gewonnenen Erkenntnisse die Leistungslücken zu schließen und durch ständige Verbesserung auf Dauer eine Spitzenposition zu bekleiden“"(Hillen 2000: 98).

Das Benchmarking dynamisiert sozusagen die Unternehmens- und Geschäftsfelderstrategien durch seine Kontinuität des Vergleichs mit dem besten Konkurrenten.

Das Benchmarking verlangt einen spezifischen Informationsverarbeitungsprozess, der bewusst über die Branchengrenzen hinweg geht. Problemgrößen sind Qualität, Kosten und Zeit: Benchmarking als Kriterium für Gästezufriedenheit, Effektivität und Effizienz, für Flexibilität und Just in Time.

\section{Umsetzungsmöglichkeiten des Lean Management im Tourismus}

Die Frage, ob sich Lean Management im Tourismus umsetzen lässt, ist aus den vorstehenden Ausführungen zweifellos zu bejahen.
In ihrer Arbeit zum „Lean Management in der Hotellerie“ kommt von Kretschmann zu folgender, auf den ganzen Tourismus anwendbaren Schlussfolgerung:, Aufgrund der dargestellten Branchenmerkmale und -situation wird es langfristig der Hotellerie unmöglich sein, sich der Einführung von Lean Management zu entziehen. Eine Anpassung der betrieblichen Kosten an die erzielbaren Zimmer- und Gastronomieverkaufspreise ist unumgänglich. Conditio sine qua non ist allerdings die erfolgreiche Bewusstseinsschaffung für die Unausweichlichkeit der notwendigen Änderungen bei den Mitarbeitern, sodass diese den dazu erforderlichen Wertewandel in gleichem Masse vollziehen. In der Hotellerie sind noch ungeahnte und enorme Effizienz- und Synergiepotentiale vorhanden, die mittels Lean Management erschlossen werden können“ (Kretschmann 1994: 8). Den Weg zum schlanken Hotel dokumentiert die Abbildung 6 auf der Folgeseite.

\section{Zusammenfassung}

Lean Management nutzt ein Bündel von Prinzipien, Konzeptionen und Maßnahmen zur effektiven und effizienten Planung, Gestaltung und Kontrolle der gesamten Wertschöpfungskette eines Unternehmens. Lean Management stellt den Menschen in den Mittelpunkt des unternehmerischen Geschehens. Zusätzlich zur schlanken Produktion kennzeichnen sich "leane“ Unternehmen durch ein besonderes Verhältnis zu den Kunden, den Lieferanten, den MitarbeiterInnen sowie durch eine besondere Finanzstrategie. Lean Management lässt sich nicht ohne grundsätzliche Überlegungen zur Unternehmenspolitik verwirklichen. Es muss daher die Zielausrichtung der Unternehmenspolitik, die eine Grundorientierung anspricht, festgelegt werden. Dies enthält übergeordnete und sektorielle Ziele als Richtziele.

$\mathrm{Zu}$ den übergeordneten Unternehmenszielen gehören qualitative Ziele wie Erhaltung der unternehm-

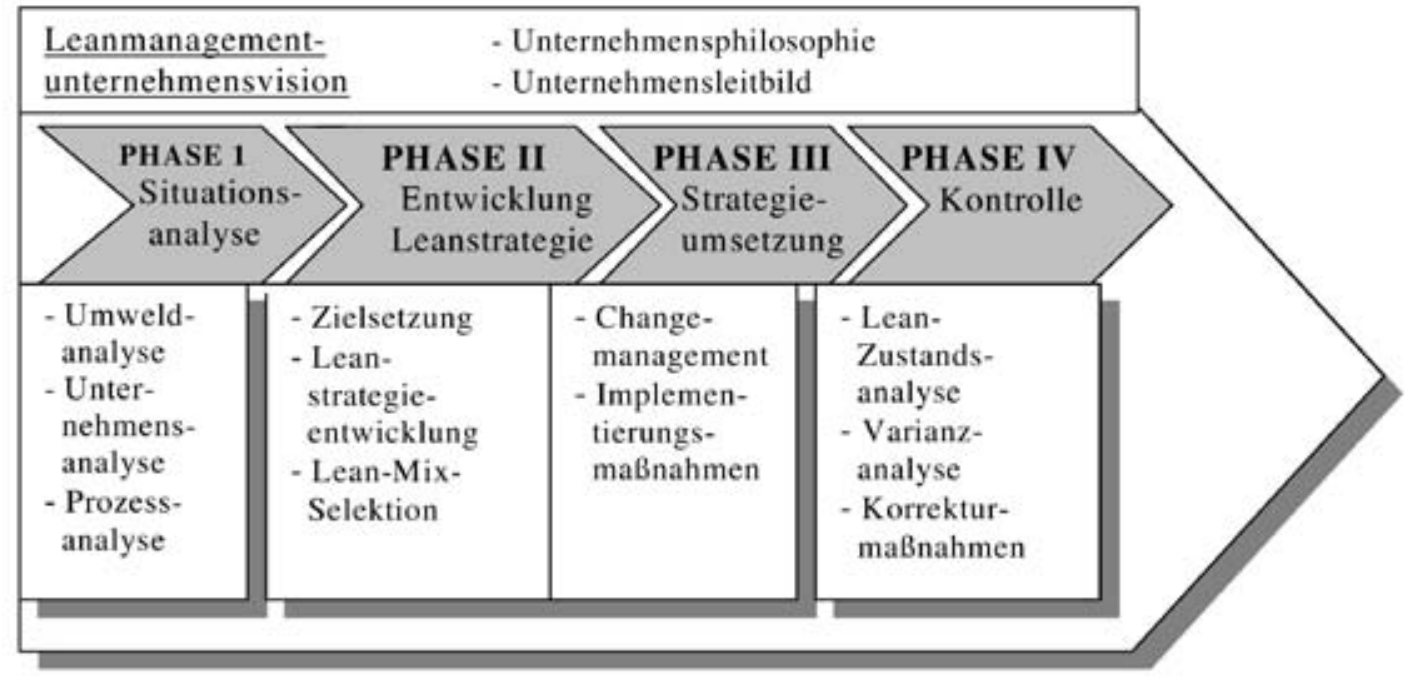

Abb. 6. Der Weg zum schlanken Hotel (Dettmer et al. 1999: 73) 
erischen Selbständigkeit oder Ausrichtung auf Sozialund Umweltverträglichkeit, und quantitative Ziele wie Sicherstellung der Rentabilität, Festlegung von Kapazitäten, Aufrechterhaltung eines vorteilhaften Preis-/ Leistungsverhältnisses usw. Sektorielle Unternehmensziele betreffen einzelne Tätigkeitsbereiche wie Marketing, Organisationsstruktur u.a.m.

Der sorgfältige Umgang mit den personellen und sachlichen Ressourcen im Lean Management geht von zentralen Denkansätzen und Leitgedanken aus:

- Proaktives Denken, auf die Zukunft des Unternehmens ausgerichtet

- Sensitives Denken, auf die Umwelt bezogen, anpassungsbereit

- Ganzheitliches Denken, das Ganze bedenken

- Potentialdenken, Erschließung aller Ressourcen (personell, sachlich, finanziell)

- Ökonomisches Denken, Vermeidung jeder Verschwendung, sparsam wirtschaften.

\section{References}

Bösenberg, D.; H. Metzen, H. 1995. Lean Management - Vorsprung durch schlanke Konzepte. Landsberg.

Bleicher, K. 2004. Das Konzept Integriertes Management. Frankfurt/New York.
Dehoga u. a. (Hrsg.). 1992. Marketing der Gastfreundschaft. Bonn/Wien/Bern/Zürich, Frankfurt.

Dettmer, H.; Hausmann, Th.; Kaspar, C.; Oppitz, W.; Schneid, W. 1999. Tourismusbetriebswirtschaft 2. Managementformen im Tourismus. Wien u.a.

Gardini, M. A. 1994. Integriertes Qualitätsmanagement in der Hotellerie, in Jahrbuch für Fremdenverkehr DWIF. München.

Hausmann, Th. 2006. Betriebswirtschaftslehre für das Gastgewerbe - managementorientiert. Hamburg.

Hillen. 2000. Benchmarking bei der Commerzbank, in M. Bruhn u.a. (Hrsg.). Dienstleistungsqualität. Wiesbaden.

Hopfenbeck, W. 2002. Allgemeine Betriebswirtschafts und Managementlehre. Landsberg.

Kaspar, C. 1995. Management im Tourismus, eine Grundlage für die Führung von Tourismusunternehmungen und organisationen. Bern/Stuttgart/Wien.

Kretschmann, C. V. 1994. Lässt sich Lean Management in der Hotellerie umsetzten? Arbeitsunterlage zum Tourismus Nr. 5, Institut für Tourismus und Verkehrswirtschaft, St. Gallen.

Pfeiffer, W.; Weiss, E. 1992. Lean Management: Grundlagen der Führung und Organisation industrieller Unternehmen. Berlin.

Romeiss-Stracke, F. 1995. Service-Qualität im Tourismus. München.

Schaetzing, E. E. 2002. Lean Management in Hotellerie und Gastronomie. Frankfurt.

\title{
LEAN MANAGEMENT - VADYBOS FORMA SU AUGANČIA SVARBA TURIZMUI
}

\author{
R. Ginevičius, T. Hausmann, ̌̌. Schafir
}

\section{Santrauka}

Integracinė optimizacija turi labai svarbią reikšmę. Kompanijos ir jos tiekejjų ištekliai bei procesai turi būti orientuoti ị vartotojų naudą taip, kad būtų išvengta nereikalingo švaistymo. Industrinėse kompanijose tai žinoma jau ilgą laiką. Straipsnyje tiriamas klausimas, kaip lean management gali būti pritaikytas kompanijose ir apgyvendinimo industrijoje.

Reikšminiai žodžiai: pagrindinès mintys, proaktyvus mąstymas, jautrus mąstymas, visuminis mąstymas, potencialus mąstymas, ekonomiškas mąstymas, pagrindinè proaktyvaus mąstymo mintis, pagrindinè jautraus mąstymo mintis, pagrindinė visuminio mąstymo mintis, pagrindinè potencialaus mąstymo mintis.

Romualdas GINEVIČIUS. Doktor habil., Professor. Rektor der Vilnius Gediminas Technical University (VGTU). Doktor (1975). Doktor habil. (1997, VGTU). Autor von mehr als 200 wissenschaftlichen Artikeln, die in Litauen und weltweit publiziert wurden sowie von zahlreichen Buchveröffentlichungen. Er ist z. B. Vorsitzender der Privatisierungskommission des Litauischen Parlamentes (Seimas), Vize-Präsident der International Academy of Informatisation. Mitglied mehrerer Akademien und des Lithuanian Committee for Research Prices.

Thomas HAUSMANN. Dipl.-Betriebswirt, MBA. Wissenschaftlicher Angestellter/Dozent. Fachhochschule Stralsund. Dem Studium der Betriebswirtschaft folgte eine Anstellung in der Holding eines großen Industrieunternehmens als Leiter der zentralen Projektorganisation; diesem Tätigkeitsfeld schloss sich die Mitgliedschaft in der zentralen Geschäftsleitung des Konzerns an. Seit 1994 angestellt in den Fachgebieten Betriebswirtschaftslehre und Tourismuswirtschaft an der Fachhochschule Stralsund. Zahlreiche Publikationen als Herausgeber und Autor auf dem Gebiet der Wirtschaftswissenschaften, insbesondere Tourismus. 
Schlomo SCHAFIR. Dr.-Ing., Dr. h.c., Professor Fachhochschule Stralsund. Studierte am Kaunas Polytechnischen Institut Elektrotechnik, an der Universität Jerusalem Philosophie, an der Technischen Universität West-Berlin Elektrotechnik, Informatik und Wirtschaft. Seit 1976 war Herr Schafir volltagsbeschäftigt in der Forschung und Entwicklung der Max Planck Gesellschaft, AEG, Siemens, Bosch und Schindler. Im Jahr 1997 wurde er für das Fach Internationales und Baltisches Management im Studiengang Baltic Management Studies der Fachhochschule Stralsund als Dozent berufen. 